\title{
OPEN Anthrax toxin translocation complex reveals insight into the lethal factor unfolding and refolding mechanism
}

\begin{abstract}
Alexandra J. Machen ${ }^{1}$, Mark T. Fisher ${ }^{1 \bowtie}$ \& Bret D. Freudenthal ${ }^{1 \bowtie}$
Translocation is essential to the anthrax toxin mechanism. Protective antigen (PA), the binding component of this AB toxin, forms an oligomeric pore that translocates lethal factor (LF) or edema factor, the active components of the toxin, into the cell. Structural details of the translocation process have remained elusive despite their biological importance. To overcome the technical challenges of studying translocation intermediates, we developed a method to immobilize, transition, and stabilize anthrax toxin to mimic important physiological steps in the intoxication process. Here, we report a cryoEM snapshot of $\mathrm{PA}_{\text {pore }}$ translocating the $\mathrm{N}$-terminal domain of $L F\left(L F_{N}\right)$. The resulting $3.3 \AA$ structure of the complex shows density of partially unfolded $L_{F}$ near the canonical $P A_{\text {pore }}$ binding site. Interestingly, we also observe density consistent with an $\alpha$ helix emerging from the $100 \AA \AA$ barrel channel suggesting LF secondary structural elements begin to refold in the pore channel. We conclude the anthrax toxin $\beta$ barrel aids in efficient folding of its enzymatic payload prior to channel exit. Our hypothesized refolding mechanism has broader implications for pore length of other protein translocating toxins.
\end{abstract}

The anthrax toxin is not only a deadly Bacillus anthracis virulence factor, but also serves as a model system of protein translocation and as a peptide therapeutic delivery platform ${ }^{1,2}$. Its biological importance and biotechnology utility have spurred significant biochemical and biophysical advances in understanding the anthrax intoxication mechanism. In order to gain entry into the cell, this archetypical AB toxin must cross the endosomal membrane. Membrane penetration is accomplished by the $\mathrm{B}$ component of anthrax toxin, termed protective antigen (PA). PA forms a translocon pore through which lethal factor (LF) or edema factor (EF), the A component, translocates. Here, we developed an approach to elucidate the structural and mechanistic details of the anthrax toxin during translocation in an effort to understand how LF unfolds in the endosome, translocates through PA, and refolds in the cytosol.

An overview of the anthrax toxin mechanism has been reviewed by the Collier lab ${ }^{1}$ and is briefly summarized here. The first step in intoxication is the $85 \mathrm{kDa}$ monomeric PA binding to host cell receptors. Then the prodomain of $\mathrm{PA}$ is cleaved leaving the $63 \mathrm{kDa} \mathrm{PA}$ to oligomerize into heptameric or octameric prepore $\left(\mathrm{PA}_{\text {prepore }}\right)^{3,4}$. $\mathrm{Up}$ to three $\mathrm{LF}$ and/or EF components can bind to the $\mathrm{PA}_{\text {prepore }}$ heptamer ${ }^{4-6}$. The $\mathrm{AB}$ toxin complex is endocytosed through clathrin mediated endocytosis ${ }^{7}$. As the endosome acidifies, $\mathrm{PA}_{\text {prepore }}$ undergoes a conformational change to a pore $\left(\mathrm{PA}_{\text {pore }}\right)^{8}$. This pore inserts into the endosomal membrane to form a channel. The low $\mathrm{pH}$ of the endosome and the $\mathrm{pH}$ gradient between the endosome and the cytosol facilitate LF or EF to unfold and rapidly translocate into the cytosol in a hypothesized Brownian ratchet mechanism ${ }^{9}$. Natively refolded LF and EF in the cytosol are then able to perform their virulent enzymatic functions ${ }^{10}$.

The overall structure of the $\mathrm{PA}_{\text {pore }}$ translocon can be divided into two regions: the funnel and the channel (Fig. 1A). The first region, the funnel, facilitates binding and unfolding of LF. LF binds to the rim of the PA funnel and is guided down the narrowing structure. The second region of $\mathrm{PA}_{\text {pore }}$ is the channel, a $\beta$ barrel that extends from the funnel and spans the endosomal membrane. Three nonspecific $P_{\text {pore }}$ clamp sites $(\alpha, \Phi$, and charge clamp) aid in the translocation of LF (Fig. 1A). The a clamp is located at the PA funnel rim, is formed by adjacent PA protomers, and binds helical portions of LF to position them towards the pore lumen. Heptameric $\mathrm{PA}_{\text {pore }}$ has seven potential a clamp binding sites. A crystal structure of the $\mathrm{N}$-terminal domain of LF $\left(\mathrm{LF}_{\mathrm{N}}\right)$ bound to the $\mathrm{PA}_{\text {prepore }}$ revealed the a clamp binding site ${ }^{11}$. Blocking of this a clamp binding site results in reduced 


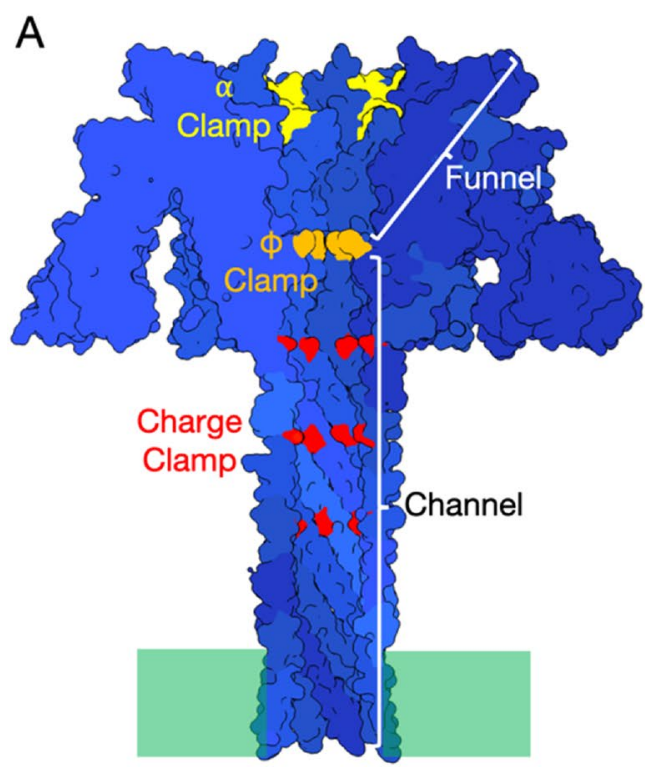

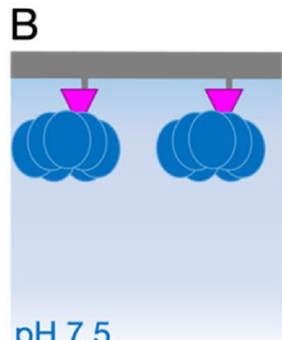

$\mathrm{pH} 7.5$

$\mathrm{E}$

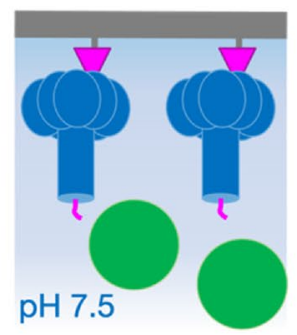

C

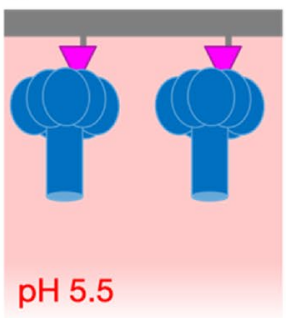

$\mathrm{F}$

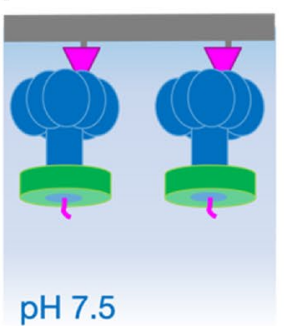

D

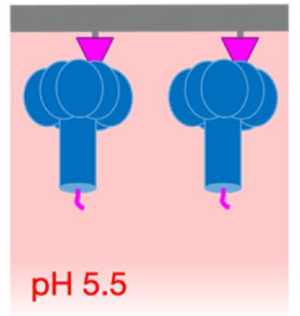

G

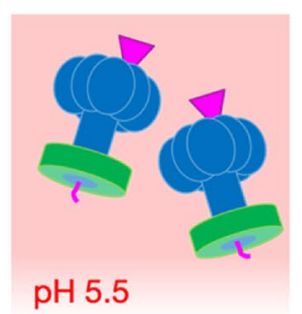

Figure 1. Anthrax toxin immobilization, translocation, and nanodisc stabilization (TITaNS) method (A) $\mathrm{PA}_{\text {pore }}$ side view slice with funnel shape from $\alpha$ clamp (yellow) to $\Phi$ clamp (orange) and charge clamp (red) inside pore $\beta$ barrel channel indicated. (B) Immobilization of $\mathrm{LF}_{\mathrm{N}}$ (magenta) $\mathrm{PA}_{\text {prepore }}$ (blue) complexes on thiol sepharose beads (grey surface). (C) $\mathrm{PA}_{\text {prepore }}$ transitioned to $\mathrm{PA}_{\text {pore }}$ (D) Predicted translocation complex of $\mathrm{LF}_{\mathrm{N}}-\mathrm{PA}_{\text {pore }}$ at low $\mathrm{pH}$. (E) Addition of pre-nanodisc micelle (green) to complex. (F) Nanodisc formation. (G) $\mathrm{LF}_{\mathrm{N}}-\mathrm{PA}_{\text {pore }^{-}}$ Nanodisc translocation complexes at $\mathrm{pH} 5.5$ on cryoEM grid.

translocation $^{12}$. The second clamp site is the $\Phi$ clamp, a ring of seven phenylalanine residues that maintain the $\mathrm{pH}$ gradient between the endosome and the cytosol ${ }^{13}$. The cryoEM structure of apo $P A_{\text {pore }}$ revealed the $\Phi$ clamp forms a narrow $6 \AA$ diameter ring ${ }^{14}$. Secondary structural elements, such as $\alpha$ helices, are too wide to fit through this narrow seal. Therefore, it is hypothesized that peptide substrates must completely unfold and refold in order to translocate through the $\mathrm{PA}_{\text {pore }}$ and enter the cytosol of the cell ${ }^{14}$. The $\Phi$ clamp also assists in the unfolding of LF as an unfolding chaperone $e^{2}$. The third clamp site, the charge clamp, is located within the $\beta$ barrel of $\mathrm{PA}_{\text {pore }}{ }^{15,16}$. The charge clamp deprotonates acidic side chains of LF and ensures unidirectional movement of the polypeptide ${ }^{15}$. Interestingly, the diameter of the $\mathrm{PA}_{\text {pore }} \beta$ barrel is large enough to accomidate an $\alpha$ helix, which would allow for initial refolding to occur inside the pore prior to LF entering the cytosol. However, it remains unclear what structural state LF is in when interacting with the charge clamp and within the $\beta$ barrel channel.

One of the many challenges in studying the anthrax toxin is that it is a dynamic membrane protein that functions under acidic conditions. Thus many questions remain, such as what path LF travels down the endosomal pore lumen from the a clamp to the $\Phi$ clamp, whether the $\Phi$ clamp adopts multiple states during translocation, and whether LF can partially refold inside the $\beta$ barrel pore. To address these questions, we developed a novel toxin immobilization, translocation, and nanodisc stabilization (TITaNS) method in combination with cryoEM to structurally characterize $\mathrm{PA}_{\text {pore }}$ translocating the $\mathrm{N}$-terminal domain of $\mathrm{LF}\left(\mathrm{LF}_{\mathrm{N}}\right)$. This approach provides unique mechanistic insight into how $\mathrm{LF}_{\mathrm{N}}$ interacts with the three clamp sites of $\mathrm{PA}_{\text {pore }}$. We observed density consistent with $\mathrm{LF}_{\mathrm{N}}$ unfolding prior to the a clamp, translocating through the dynamic $\Phi$ clamp, and beginning to refold in the channel of the $\mathrm{PA}_{\text {pore. }}$

\section{Results}

Assembly of anthrax translocation complexes. In vivo, the anthrax toxin undergoes a prepore to pore conformational change under acidic conditions. Previous structural studies have generally used urea to avoid aggregation during the transition from $\mathrm{PA}_{\text {prepore }}$ to $\mathrm{PA}_{\text {pore }}{ }^{17-22}$. These approaches have limitations in that they do not account for the low $\mathrm{pH}$ electrostatic microenvironment in the pore lumen predicted to be important for LF-PA interactions ${ }^{23}$ and they assume similar outcomes for chaotrope and acid induced unfolding. In order to overcome these limitations we have developed a novel assembly method for toxin immobilization, translocation, and nanodisc stabilization (called TITaNS, Fig. 1B-G). TITaNS was designed to mimic important low pH physiological states during the anthrax intoxication mechanism. This approach allows for endosomal $\mathrm{pH}$ pore formation and imaging of individual complexes in a lipid bilayer in the biologically relevant low $\mathrm{pH}$ environment ${ }^{17,24}$. TITaNS can be used in combination with techniques other than cryoEM, including mass spectrometry, nuclear magnetic resonance, surface plasmon resonance, and biolayer interferometry. TITaNS also has the potential to be adapted to screen prospective pharmaceuticals that arrest or prevent endosomal membrane insertion ${ }^{24}$.

Reversible immobilization was key to the TITaNS methodology, because it allowed the stabilized complexes to be released into solution. We began with recombinantly purified, soluble forms of $\mathrm{LF}_{\mathrm{N}}$ and $\mathrm{PA}_{\text {prepore }}$ that were mixed together in solution. The binary complex of $\mathrm{LF}_{\mathrm{N}}$ bound to $\mathrm{PA}_{\text {prepore }}$ was then immobilized onto thiol

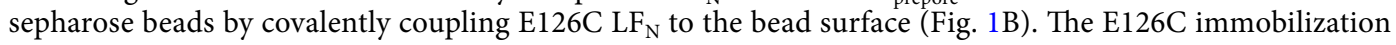


site is opposite the PA binding site. The rationale for choosing this residue is that it allows for immobilization of $\mathrm{LF}_{\mathrm{N}}-\mathrm{PA}_{\text {prepore }}$ complexes without hindering $\mathrm{PA}_{\text {prepore }}$ binding (Fig. 1B) and facilitates prepore to pore extension away from the bead surface when the complex is exposed to low $\mathrm{pH}$ (Fig. 1C). We predict this low $\mathrm{pH}$ environment initiates translocation of $\mathrm{LF}_{\mathrm{N}}$ through $\mathrm{PA}_{\text {pore }}$ in vitro (Fig. 1D). We base this prediction on computational and experimental evidence of early translocation events induced by low $\mathrm{pH}$. Specifically, molecular simulations of anthrax toxin early translocation events predict the events are strongly influenced by the protonation state of LF and are highly favorable at low $\mathrm{pH}^{23}$. Also at low $\mathrm{pH}$, the partial translocation of LF has been observed in planar lipid bilayers ${ }^{9}$. After pore formation, the next step in TITaNS was the stabilization of $\mathrm{LF}_{\mathrm{N}}-\mathrm{PA}_{\text {pore }}$ translocation complexes using nanodisc technology ${ }^{25-27}$. Pre-nanodisc micelles were added to the bead slurry and associated with the transmembrane portion of $\mathrm{PA}_{\text {pore }}$ (Fig. 1E). To promote lipid bilayer formation, we dialyzed away excess detergent (Fig. $1 \mathrm{~F})$. The soluble $\mathrm{LF}_{\mathrm{N}}-\mathrm{PA}_{\text {pore }}$-nanodisc complexes were then eluted off the thiol sepharose beads using the reducing agent dithiothreitol. Eluted complexes were transferred to the cryoEM grid and the $\mathrm{pH}$ was dropped to $\mathrm{pH} 5.5$ to capture the complex at low $\mathrm{pH}$ prior to blotting and plunge freezing (Fig. 1G). To prevent aggregation and migration of the nanodiscs to the air-water interface, we plunge froze the grids within $30 \mathrm{~s}$ of sample application. Using our TITaNS methodology, we obtained a $3.3 \AA$ reconstruction of $\mathrm{LF}_{\mathrm{N}}$ translocating through $\mathrm{PA}_{\text {pore }}$ (Fig. 2A-D).

Comparison of $\mathrm{LF}_{N}$ before and during translocation. Prior to translocation, $\mathrm{LF}_{\mathrm{N}}$ is bound to the cap of $\mathrm{PA}$ at the interface of two PA protomers with helix $\alpha 1$ bound to the $\alpha$ clamp $^{11,18}$. Our reconstruction shows a loss of $\mathrm{LF}_{\mathrm{N}}$ density in the canonical binding site above the $\mathrm{PA}_{\text {pore }}$ (Fig. 2A,B). Lethal factor translocates through $\mathrm{PA}_{\text {pore }}$ from the $\mathrm{N}$-terminus to the C-terminus. Notably, our reconstruction does not show density for residues $50-135$ in the canonical folded $L_{\mathrm{N}}$ position (Fig. 2B,D) ${ }^{11,17}$. However, density for residues 136-250 are apparent. Our model of unfolding, translocating $\mathrm{LF}_{\mathrm{N}}$ is consistent with a molten globular intermediate state at low $\mathrm{pH}^{28}$.

Molecular interactions of translocation complex. In addition to a loss of $\mathrm{LF}_{\mathrm{N}}$ density in the canonical binding site above the $\mathrm{PA}_{\text {pore }}$, our reconstruction shows added density inside the pore lumen indicating a translocating complex (Fig. 3). This translocating $\mathrm{LF}_{\mathrm{N}}$ density is discontinous. Dashed lines are used to indicate regions where there is a not enough density to empirically build model (Fig. 3A). Density near the top of the $\mathrm{PA}_{\text {pore }}$ funnel was in proximity to several hydrophobic residues of $\mathrm{PA}_{\text {pore }}$ in the a clamp (Fig. 3B). F202, F236, and F464 have previously been shown to be important for early translocation. Specifically, mutagenesis of F202 and F236 decreases translocation ${ }^{16}$ while F464 has shown plasticity when binding translocation substrates ${ }^{18}$. These hydrophobic residues are predicted to aid in unfolding LF and stabilizing unfolded intermediates as they transition into the pore ${ }^{11}$. Our results are consistent with F202, F236, and F464 facilitating translocation of LF $_{\mathrm{N}}$ into the pore and toward the $\Phi$ clamp in an unfolded state. We also observed added asymmetric density in and around the $\Phi$ clamp (Fig. $3 \mathrm{C}$ ) that was not visible when we compared it to the previously published apo $\mathrm{PA}_{\text {pore }}$ cryoEM structure $^{14}$. Specifically, there is density in the center of the $\Phi$ clamp (Fig. 3C). We attribute this density to unfolded $\mathrm{LF}_{\mathrm{N}}$ interacting with the benzyl rings of the $\mathrm{PA}_{\text {pore }} \Phi$ clamp as $\mathrm{LF}_{\mathrm{N}}$ is translocating through the pore. In addition, the density for each of the $\mathrm{PA}_{\text {pore }} \mathrm{F} 427$ residues was smeared in plane with the benzyl ring suggesting rotameric states moving up and down (Fig. 3D).

Translocating $\mathrm{LF}_{\mathrm{N}}$ density was also observed in the $\beta$ barrel of the $\mathrm{PA}_{\text {pore. }}$. Focused refinement of the $\beta$ barrel interior revealed density consistent with $\alpha$ helices along with portions of unfolded peptide (Fig. 3E-G). Notably, density located at the $\mathrm{PA}_{\text {pore }}$ charge clamp is consistent with an a helix and suggests the deprotonated state of LF favors helix formation within the pore. Canonical charge clamp residues D276, E343, and D335 are shown in Fig. $3 \mathrm{E}$ with the predicted $\mathrm{LF}_{\mathrm{N}}$ density translocating through the center of the channel. Further down the pore we observe density for helix al emerging from the channel exit (Fig. 3F,G). Our results provide evidence for initial refolding of LF secondary structure both inside and upon exit from the $\mathrm{PA}_{\text {pore }}$. These results are consistent with ribosome exit tunnel studies that, using optical tweezers and molecular dynamics simulations, showed excluded volume effects and electrostatic interactions contribute to substrate folding ${ }^{29}$. In the future, TITaNS could be combined with site specific cross-linking between the $\mathrm{PA}_{\text {pore }}$ clamp sites and $\mathrm{LF}_{\mathrm{N}}$ to capture other unfolded, translocating intermediates.

Next, we wanted to determine whether there were any differences between $\mathrm{PA}_{\text {pore }}$ protomers that interacted directly with the translocating $\mathrm{LF}_{\mathrm{N}}$ compared to protomers that did not interact with translocating $\mathrm{LF}_{\mathrm{N}}$. Importantly, symmetry operations were not used during single particle analysis. To compare the $\mathrm{PA}_{\text {pore }}$ protomers, we aligned the seven protomer chains that compose the $\mathrm{PA}_{\text {pore }}$ (Fig. S1A). Comparison of the chains showed little difference in the backbone or side chain rotamers for the majority of the residues at the $\mathrm{LF}_{\mathrm{N}}-\mathrm{PA}_{\text {pore }}$ interfaces as well as the protomer-protomer interfaces. This rigidity is likely necessary for the $\mathrm{PA}_{\text {pore }}$ to maintain a stable $\beta$ barrel and perform its function under endosomal conditions. During this analysis, we also examined the conformation of the receptor binding domain of $\mathrm{PA}_{\text {pore. }}$. The receptor binding domain is connected to the main body of $\mathrm{PA}_{\text {pore }}$ by a single loop and is responsible for anchoring the toxin to the host cell membrane prior to complex endocytosis and pore formation ${ }^{30}$. Interestingly, the receptor binding domain did show different conformations for each protomer and indicates a degree of conformational flexibility (Fig. S1A-C). This variability between receptor binding domains likely arose from the acidic conditions and lack of a receptor. Overlaying our $\mathrm{PA}_{\text {pore }}$ chains with the crystal structure of $\mathrm{PA}_{\text {prepore }}$ bound to its receptor, capillary morphogenesis protein 2 (CMG2), revealed several conformations not conducive to receptor binding (Fig. S1C). Specifically, PA E194 was not in position to form the metal ion-dependent adhesion site (MIDAS) motif in the receptor binding pocket ${ }^{31}$. This indicates that without a receptor bound, the receptor binding domain loops adopt multiple states. 

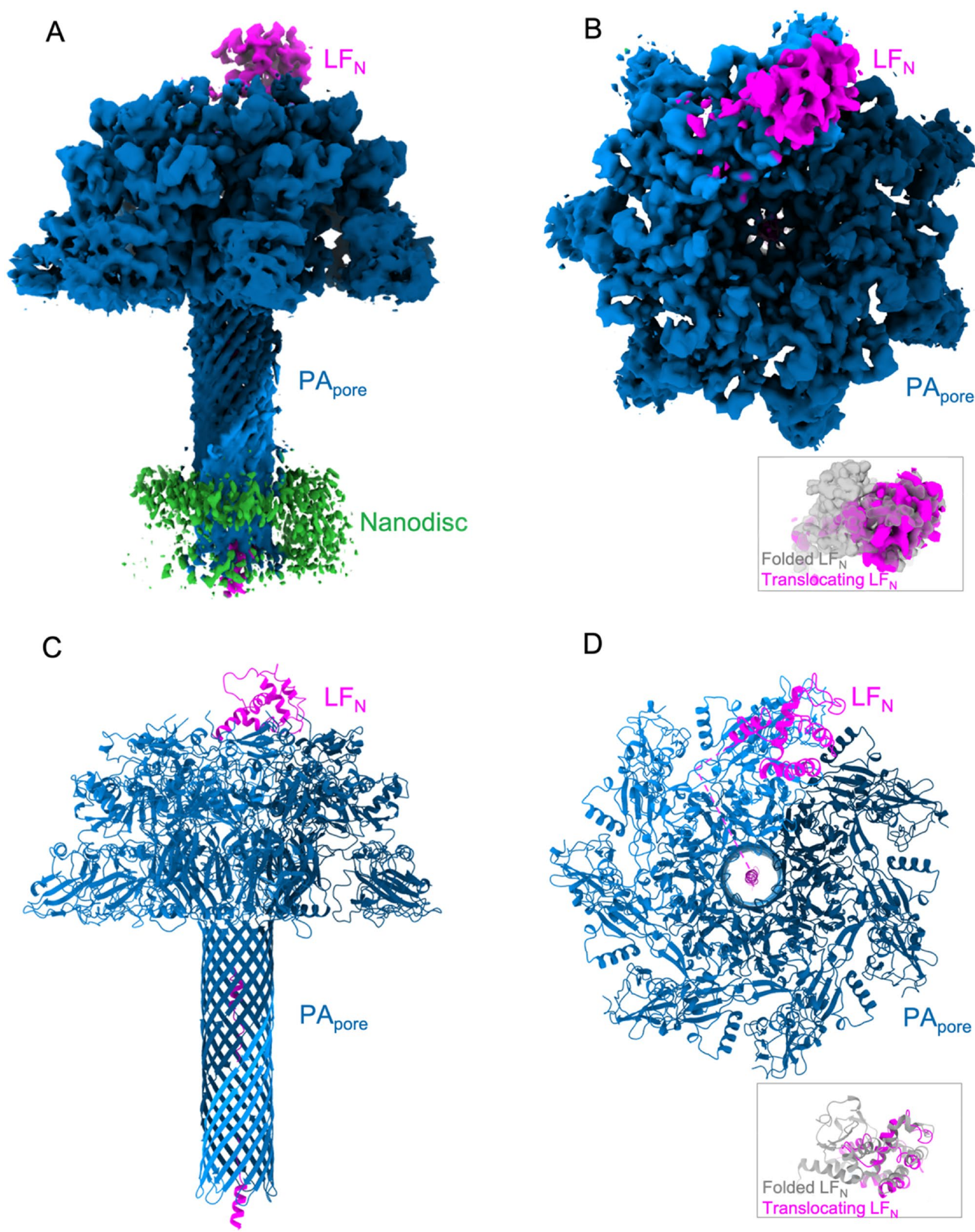

D

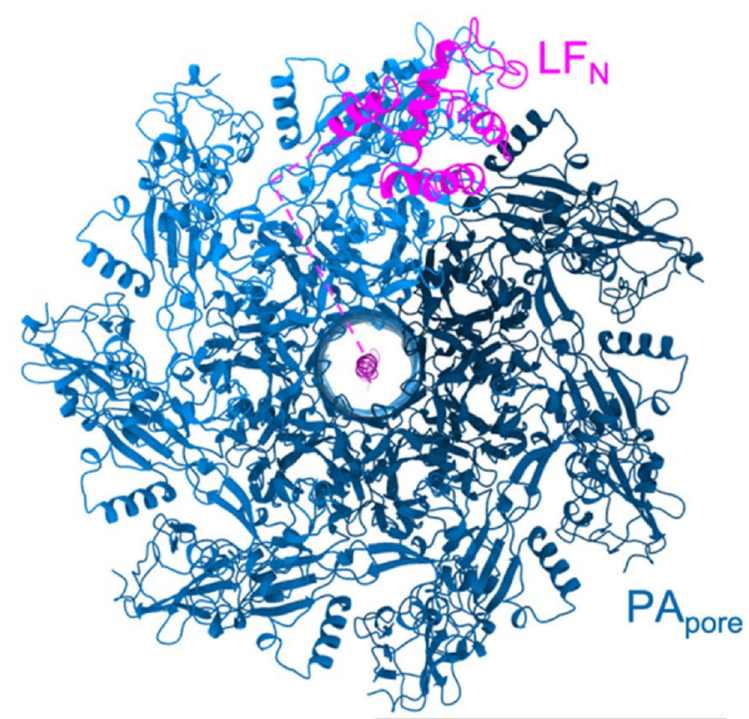

Figure 2. Overview of the anthrax toxin translocation complex. CryoEM density map (A) side and (B) top view of $\mathrm{LF}_{\mathrm{N}}$ translocating through $\mathrm{PA}_{\text {pore }}$. Inset: folded $\mathrm{LF}_{\mathrm{N}}$ model density (PDB 6PSN) compared to translocating $\mathrm{LF}_{\mathrm{N}}$ density. Molecular model $(\mathbf{C})$ side and (D) top view of $\mathrm{LF}_{\mathrm{N}}$ translocating through $\mathrm{PA}_{\text {pore }}$. Inset: folded $\mathrm{LF}_{\mathrm{N}}$ model (PDB 6PSN) compared to translocating $\mathrm{LF}_{\mathrm{N}}$ model. Discontinuous density indicated in model with dashed magenta lines. 

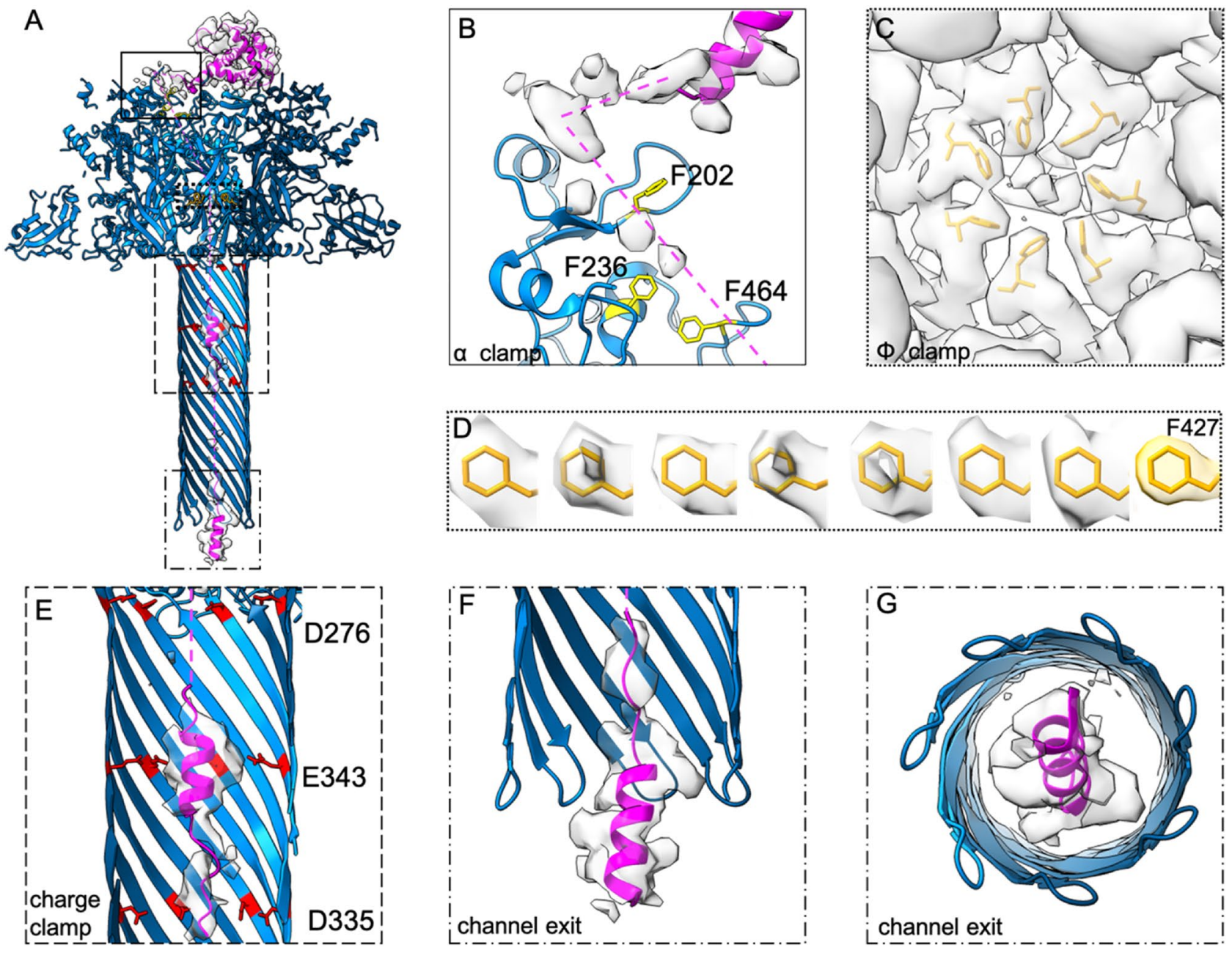

Figure 3. Unfolding and refolding of $\mathrm{LF}_{\mathrm{N}}$ at key clamp sites during translocation through $\mathrm{PA}_{\text {pore }}$ (A) Model of $\mathrm{PA}_{\text {pore }}$ (blue) translocating $\mathrm{LF}_{\mathrm{N}}$ (magenta) with a clamp, $\Phi$ clamp, and charge clamp residues shown in yellow, orange, and red respectively. CryoEM density of $\mathrm{LF}_{\mathrm{N}}$ shown in transparent grey. (B) Hydrophobic residues predicted to facilitate unfolding in early translocation from a clamp to $\Phi$ clamp. (C) $\Phi$ clamp ring with $\mathrm{LF}_{\mathrm{N}}$ density. (D) F427 residues for each subunit with associated cryoEM density compared to modelled density. (E) $\mathrm{LF}_{\mathrm{N}}$ model and density in $\beta$ barrel charge clamp. (F) $\mathrm{PA}_{\text {pore }}$ channel exit into the cytosol with $\mathrm{LF}_{\mathrm{N}} \alpha 1$ helical model and cryoEM density. (G) $90^{\circ}$ rotation of F. Discontinuous density indicated in model with dashed magenta lines.

\section{Discussion}

The anthrax toxin $\mathrm{PA}_{\text {pore }}$ unfolds, translocates, and refolds LF, it's enzymatic substrate. Three clamp sites aid in peptide translocation: the a clamp, the $\Phi$ clamp, and the charge clamp. We report here, cryoEM density consistent with nascent polypeptide chain translocating the length of $\mathrm{PA}_{\text {pore }}$. In our model, $\mathrm{LF}_{\mathrm{N}}$ can be seen unfolding prior to the a clamp, passing through the dynamic $\Phi$ clamp, and refolding in the $\beta$ barrel channel. This model is consistent with the hypothesis that LF needs to completely unfold in order to translocate. However, if the entire $90 \mathrm{kDa}$ enzyme were to unfold at once, deleterious folded intermediates or aggregates would likely block the $\mathrm{PA}_{\text {pore }}$ translocon, especially when multiple LF are bound to PA. Therefore, in order to efficiently translocate and refold, $\mathrm{LF}$ unfolds from the $\mathrm{N}$ to $\mathrm{C}$ terminus ${ }^{32}$. While the low $\mathrm{pH}$ of the endosome destabilizes the enzyme, it does not completely unfold into its primary sequence ${ }^{28,33}$. Our results are consistent with molten globular translocation intermediates of LF being destabilized in the acidic environment of the endosome ${ }^{28}$ with the a clamp then able to apply additional unfolding force on the protein and funnel LF towards the $\Phi$ clamp ${ }^{2}$. Translocation requires stepwise unfolding and stabilization of the unfolded intermediates to prevent aggregation. When LF binds to $\mathrm{PA}_{\text {pore}}$, helix $\alpha 1$ of LF moves away from the main body of LF and binds to the $\alpha$ clamp of $P_{\text {pore }}{ }^{11}$. From here, LF has multiple paths it could take through the $\mathrm{PA}_{\text {pore }}$ funnel, gated by the $\Phi$ clamp. We were unable to resolve distinct unfolded $\mathrm{LF}_{\mathrm{N}}$ density passed the a clamp to the $\Phi$ clamp. However, our results do suggest a favorable path from the a to the $\Phi$ clamp would involve a series of hydrophobic residues that are amenable to unfolded translocation intermediates and likely serve as checkpoints to verify the unfolded state of LF prior to the $\Phi$ clamp (Fig. 3A-B).

The $\Phi$ clamp plays a crucial roll in translocation by acting as a hydrophobic seal between the endosome and cytosol $^{9}$. Steric clashing between the narrow $\Phi$ clamp and bulkier LF side chains suggests the need for movement of the clamp ${ }^{34}$. Indeed, the dynamic nature of the $\Phi$ clamp has recently been measured using ${ }^{19} \mathrm{~F} \mathrm{NMR}^{34}$. The 
smeared density in our structure is indicative of a dynamic clamp. Our density does not imply dilation of the clamp ${ }^{35}$, so much as a up and down motion along the pore axis. This motion could be conserted or individual F427 residues moving to accommodate various translocating side chains. The compressive and tensile forces generated by the unfolding LF in the $\mathrm{PA}_{\text {pore }}$ funnel above and the refolding LF in the $\beta$ barrel channel below may also contribute to this movement. However, too much flexibility or dilation would cause the seal at the $\Phi$ clamp to be lost. Therefore, this dynamic motion must still maintain the $\mathrm{pH}$ gradient between the endosome and cytosol, while accomidating any side chain, ensuring efficient translocation. Multiple $\Phi$ clamp states have been hypothesized at $\mathrm{pH} 5.5^{35}$. Our analysis did not reveal multiple distinct states, though it does not exclude the possibility. A larger dataset or different conditions may be needed to address this question.

Helix formation inside the $\mathrm{PA}_{\text {pore }} \beta$ barrel has been hypothesized but, to our knowledge, never observed ${ }^{14,28}$. We report here, evidence of concomitant $\alpha$ helix formation and translocation inside the $\beta$ barrel of the $\mathrm{PA}_{\text {pore }}$ (Fig. 3E-G). We hypothesized that, along with changing the charge state of the peptide substrate, the charge clamp allows for a local folding environment within the $\mathrm{PA}_{\text {pore. }}$. Helical portions of LF have previously been shown to dock into the a clamp, with the periodicity of these helices aiding in efficient unfolding of $\mathrm{LF}^{36}$. We predict this periodicity is also important for hypothesized refolding of LF, beginning at the charge clamp. Our hypothesis is consistent with other anthrax toxin substrates, such as $\mathrm{LF}_{\mathrm{N}}$ fused to the catalytic chain of diphtheria toxin $\left(\mathrm{LF}_{\mathrm{N}}-\mathrm{DTA}\right)$, which did not evolve to fold in the $\mathrm{PA}_{\text {pore }}$ channel. Interestingly, these non-native substrates require chaperones for enzymatic activity ${ }^{37}$ indicating the DTA portion of these proteins do not form helices in the PA channel at optimal intervals. Our model is also reminesant of the ribosome, where helix folding in the exit tunnel aids in co-translational folding of native proteins ${ }^{38}$. We predict helix folding in the $\mathrm{PA}_{\text {pore }} \beta$ barrel aids in co-translocational folding by temporally altering LF emersion from the tunnel allowing regions to fold into tertiary structures.

A proposed unfolding-refolding translocation model is shown in Fig. $4 \mathrm{~A}$ starting with $\mathrm{LF}_{\mathrm{N}}$ bound to the funnel rim of $\mathrm{PA}_{\text {pore }} \mathrm{LF}_{\mathrm{N}}$ is unfolded and funnelled towards the $\Phi$ clamp, aided by hydrophobic residues along the funnel slope. $\mathrm{LF}_{\mathrm{N}}$ acidic residues are protonated in the acidic environment of the funnel. Completely unfolded $\mathrm{LF}_{\mathrm{N}}$ then passes the $\Phi$ clamp. This ring of F427 residues remains restrictive enough to maintain a seal while accommodating translocation. As the channel widens in the charge clamp, acidic residues are deprotonated. Folding of $\alpha$ helical portions places mechanical force on the translocating peptide, contributing to efficient translocation, and overcoming local energy minimum that could otherwise stall the complex. The newly formed, secondary structure favors unidirectional translocation by discouraging retrograde transfer through the narrow $\Phi$ clamp resulting in natively folded LF in the host cell cytosol.

Our results have implications for other toxins. PA F427 is equivalent to F454 of Clostridium perfringens iota toxin $^{39}$, F428 of Clostridium botulinum C2II binary toxin ${ }^{40}$, and W318 of Vibrio cholerae cytolysin ${ }^{41}$. We hypothesize a dynamic hydrophobic seal model is a common mechanism, applicable to these other toxins. Initial refolding in the pore channel is also likely not unique to the anthrax toxin. Indeed, other translocons, such as the iota toxin and toxin complex (Tc) toxin (Fig. 4B), have pores that extend well passed the membrane bilayer ${ }^{42,43}$. We predict these pore forming toxins have evolved extended pores to faciliate substrate refolding inside the translocon for effective intoxication. Not all pore forming toxins translocate proteins in vivo. Some, like Vibrio cholerae cytolysin (VCC) and Staphylococcus aureus a-hemolysin, form pores to distrupt ion concentrations ${ }^{41,44}$. The pore length of these toxins is noticeably shorter (Fig. 4B).

\section{Materials and methods}

Protein expression and purification. Proteins were purified as previously described ${ }^{17}$. Briefly, His $_{6}-\mathrm{SUMO}-\mathrm{LF}_{\mathrm{N}}$ E126C was expressed in BL21 cells, purified using anion exchange, and cleaved by small ubiquitin-related modifier protease ${ }^{45}$. Recombinant wild-type $\mathrm{PA}_{83}$ was expressed in the periplasm of Escherichia coli BL21 (DE3) and purified by ammonium precipitation and anion exchange chromatography ${ }^{8}$. After trypsin activation ${ }^{45}, \mathrm{PA}_{63}$ heptameric prepores were formed using anion exchange and size exclusion chromatography. Membrane scaffold protein 1D1 (MSP1D1) was expressed from the pMSP1D1 plasmid (AddGene) with an $\mathrm{N}$-terminal His-tag and was purified by affinity chromatography ${ }^{27}$.

LF $_{N}$-PA-nanodisc complex formation for CryoEM with TITaNS. We have previously dealt with heterogenous sample preparations of one, two, and three $\mathrm{LF}_{\mathrm{N}}$ bound to $\mathrm{PA}_{\text {pore }}$. We found incubating $\mathrm{LF}_{\mathrm{N}}$ with $\mathrm{PA}_{\text {prepore }}$ at sub-stoichiometric ratios prior to $\mathrm{LF}_{\mathrm{N}}$ immobilization and $\mathrm{PA}$ pore formation resulted in more homogenous samples ${ }^{17}$. For this work, E126C $\mathrm{LF}_{\mathrm{N}}$ and $\mathrm{PA}_{\text {prepore }}$ were incubated in solution at a ratio of 1:2, respectively. Complexes were then immobilized by coupling E126C $\mathrm{LF}_{\mathrm{N}}$ to activated thiol sepharose $4 \mathrm{~B}$ beads (GE Healthcare Bio-Sciences, Pittsburgh, PA, USA) in Assembly Buffer (50 mM Tris, $50 \mathrm{mM} \mathrm{NaCl,} 10 \mathrm{mM} \mathrm{CaCl}_{2} \mathrm{pH}$ 7.5) at $4{ }^{\circ} \mathrm{C}$ for $12 \mathrm{~h}$. Beads were washed three times with Assembly Buffer to remove any unbound $\mathrm{PA}_{\text {prepore. The }}$ immobilized $\mathrm{LF}_{\mathrm{N}}-\mathrm{PA}_{\text {prepore }}$ complexes were then incubated in low $\mathrm{pH}$ buffer $(10 \mathrm{mM}$ acetate, $50 \mathrm{mM}$ Tris, $50 \mathrm{mM}$ $\mathrm{NaCl}, 10 \mathrm{mM} \mathrm{CaCl}_{2} \mathrm{pH}$ 5.5) to transition the $\mathrm{PA}_{\text {prepore }}$ to $\mathrm{PA}_{\text {pore }}$ and initiate translocation of $\mathrm{LF}_{\mathrm{N}}$. Our previous cryoEM work used urea instead of low $\mathrm{pH}$ to transition $\mathrm{PA}_{\text {prepore }}$ to $\mathrm{PA}_{\text {pore }}{ }^{17}$. The beads were then washed in Assembly Buffer at neutral $\mathrm{pH}$ three times. Next, pre-nanodisc micelles (2.5 $\mu \mathrm{M}$ MSP1D1, $97.5 \mu \mathrm{M}$ 1-palmitoyl2-oleoyl-sn-glycero-3-phosphocholine (POPC) (Avanti, Alabaster, AL, USA), 65 (POPG) in 25 mM Na-cholate (Sigma-Aldrich, St. Louis, MO, USA), $50 \mathrm{mM}$ Tris, and $50 \mathrm{mM} \mathrm{NaCl}$ ) were added and bound to the aggregationprone hydrophobic transmembrane $\beta$-barrel of $\mathrm{PA}_{\text {pore }}$. The micelles were collapsed into nanodiscs by removing Na-cholate using dialysis with Bio-Beads (BIO RAD, Hercules, CA, USA). Stabilized complexes were released from the thiol sepharose beads by reducing the $\mathrm{E} 126 \mathrm{C} \mathrm{LF}_{\mathrm{N}}$-bead disulfide bond using $50 \mathrm{mM}$ dithiothreitol (DTT) (Goldbio, St. Louis, MO, USA) in Assembly Buffer. Assembled complexes were initially confirmed using 


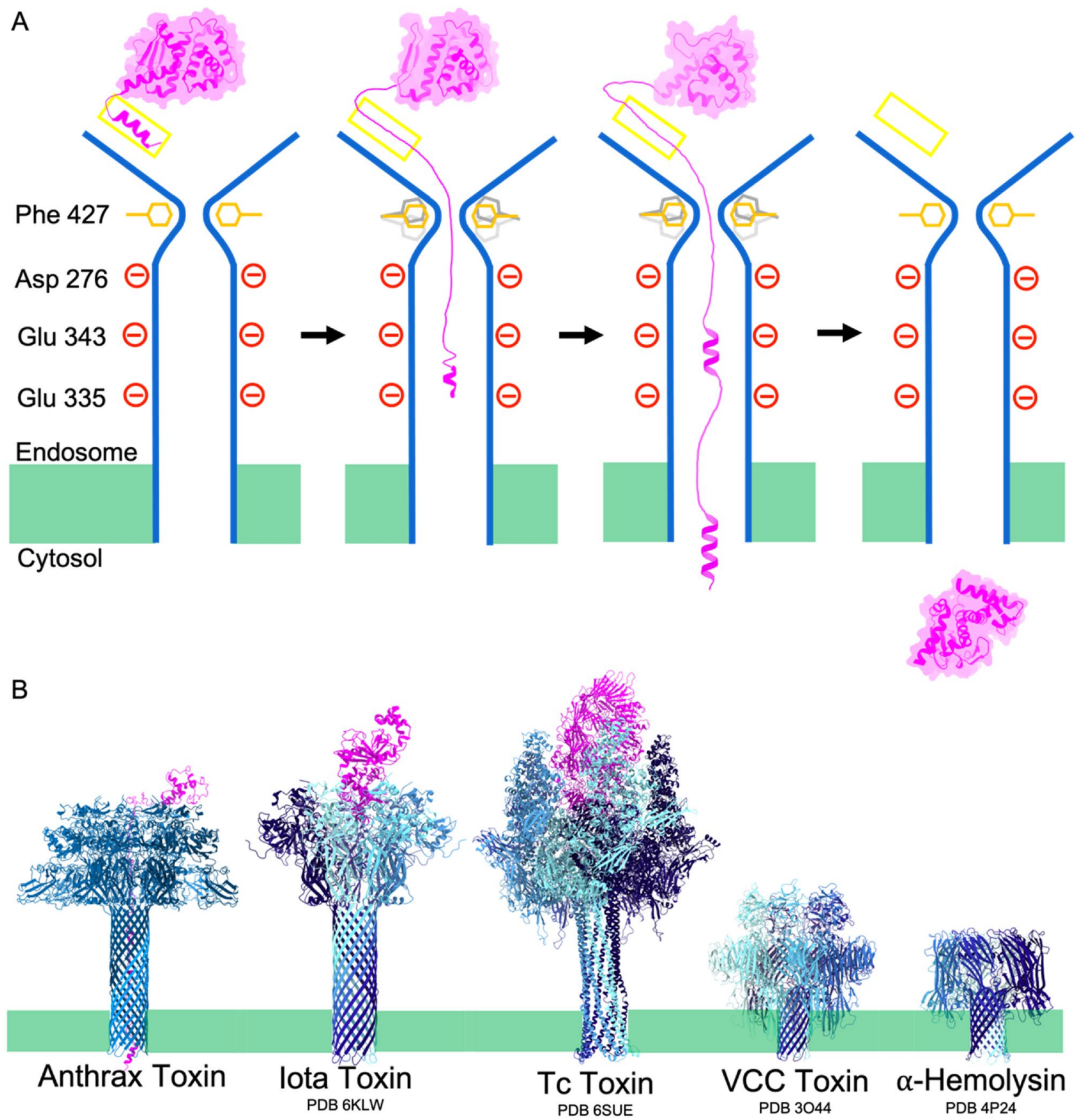

Figure 4. Proposed $\mathrm{AB}$ toxin extended pore refolding mechanism. (A) Unfolding-refolding translocation model of anthrax toxin. $\mathrm{LF}_{\mathrm{N}}$ (magenta) translocates through $\mathrm{PA}_{\text {pore }}$ (blue) passing the a clamp (yellow), the $\Phi$ clamp (orange and grey), and the charge clamp (red). Helical portions of $\mathrm{LF}_{\mathrm{N}}$ begin to refold in the channel ensuring proper tertiary refolding of $\mathrm{LF}_{\mathrm{N}}$. (B) Comparison of toxin pore length between toxins that translocate proteins vs toxins that disrupt ion gradients. Membrane bilayer represented in green.

negative-stain TEM. Complexes were stored at $-80^{\circ} \mathrm{C}$ prior to cryoEM grid preparation. Complex formation has also previously been confirmed using mass spectrometry and biolayer interferometry ${ }^{46}$.

Grid preparation for CryoEM. Complexes stored at $-80^{\circ} \mathrm{C}$ were thawed on ice. A glow discharged Quantifoil R1.2/1.3 300 M Cu holey carbon grid was placed inside the FEI Vitrobot Mark IV humidity chamber at 100\% humidity. Then, $2 \mu \mathrm{l}$ of thawed sample was applied to the grid followed by $0.5 \mu \mathrm{L}$ of $1 \mathrm{M}$ acetate $\mathrm{pH} 5.5$. The grids were then blotted and plunge frozen in liquid ethane. Frozen grids were stored in liquid nitrogen prior to use.

CryoEM data collection and image processing. CryoEM grids were loaded into a FEI Titan Krios electron microscope operated at $300 \mathrm{kV}$ for automated image acquisition with serialEM ${ }^{47}$. cryoEM micrographs were recorded as movies on a Gatan K2 Summit direct electron detection camera using the electron counting 
mode in super resolution mode at $\times 130 \mathrm{~K}$ nominal magnification, a pixel size of $0.535 \AA$ A per pixel, and defocus ranging between -1 and $-3 \mu \mathrm{m}$. Total dose was $50.76 \mathrm{e}^{-} / \AA^{2}$. Total exposure time was $9 \mathrm{~s}$ and fractionated into 45 frames with $200 \mathrm{~ms}$ exposure time for each frame. In total, 6,515 micrographs were taken in a continuous session. Frames in each movie were aligned and averaged for correction of beam-induced drift using MotionCor2 and cryoSPARC patch motion correction to generate a micrograph ${ }^{48,49}$. Micrographs generated by averaging all frames of each movie were used for defocus determination and particle picking. Micrographs obtained by averaging frames 2-36 (corresponding to $\sim 40 \mathrm{e}^{-} / \AA^{2}$ ) were used for two- and three-dimensional image classifications. The best 4,488 micrographs were selected for the following in-depth data processing.

Single particle analysis and density modification. Single particle analysis was performed using cryoSPARC v2.15 $5^{48}$ (Fig. S2). A random subset of micrographs was selected for blob particle picking. These particles were subjected to $2 \mathrm{D}$ classification in order to obtain a set of five particle templates. Using these templates, 2,076,581 particles were selected from 4,488 micrographs. After multiple rounds of 2D classification, the remaining 671,090 'good' particles were used to create an ab initio model. Heterogenous classification with four classes was then performed and the class with full length $\beta$ barrel and distinct nanodisc was selected. To select particles with $\mathrm{LF}_{\mathrm{N}}, 3 \mathrm{D}$ variability analysis was performed with three orthogonal principle modes (i.e. eigenvectors of $3 \mathrm{D}$ covariance) and a mask of $\mathrm{LF}_{\mathrm{N}}$ bound in each of the seven possible binding sites filtered to $30 \AA$ and gaussian blurred. 122,651 particles from three resulting clusters with potential $\mathrm{LF}_{\mathrm{N}}$ density were selected for further processing. A homogenous refinement on the per particle motion and CTF corrected particles was performed resulting in a $3.3 \AA$ cryoEM density map. Resolution was determined using gold standard Fourier shell correlation with a cut off of 0.143 . Next, local refinements and density modifications were performed (Fig. S3). To further characterize bound $\mathrm{LF}_{\mathrm{N}}$, local refinement of the cap of the $\mathrm{PA}_{\text {pore }}$ was performed using a mask of $\mathrm{LF}_{\mathrm{N}}$ (PDB 3KWV) low pass filtered to $30 \AA$. For the $\beta$ barrel interior, a cylindrical mask was used. Phenix density modification was performed on the homogenous refinement and local refinement half maps to further improve the density for $\mathrm{PA}_{\text {pore }}$ and $\mathrm{LF}_{\mathrm{N}}$, respectively ${ }^{50}$. Phenix combine focused maps was then used to create a composite map (Fig. S3) ${ }^{51}$. The map showed density surrounding the transmembrane region of the beta barrel that we interpret as nanodisc density. As has been reported previously ${ }^{14}$, there was also disordered density surrounding the outside of the middle of the $\beta$ barrel. This additional density was masked out of the final map using a $30 \AA$ mask of $\mathrm{LF}_{\mathrm{N}}-\mathrm{PA}_{\text {pore }}$ inserted into a nanodisc.

Model building and refinement. An initial model using PDB 6PSN was docked into the cryoEM map using Chimera map to model ${ }^{52}$. The $\mathrm{LF}_{\mathrm{N}}$ coarse model was adjusted manually using $\operatorname{Coot}^{53}$ to fit the density starting at the C-terminus. Model $\alpha$ helical assignments were based on helicity in original model, cryoEM density diameter, and consistency with previously published helical density. The $\mathrm{PA}_{\text {pore }}$ coarse model was refined using PHENIX real space refine ${ }^{51}$. Individual atomic model side chains were manually adjusted to fit the density map using $\operatorname{Coot}^{53}$. This process was repeated iteratively until an optimal model was obtained. Ramachandran plots and MolProbity ${ }^{54}$ were used to assess model quality. Supplementary Table 1 is a summary of cryoEM data collection and processing as well as model building and validation.

Received: 29 January 2021; Accepted: 27 May 2021

Published online: 22 June 2021

\section{References}

1. Young, J. A. \& Collier, R. J. Anthrax toxin: Receptor binding, internalization, pore formation, and translocation. Annu. Rev. Biochem. 76, 243-265 (2007).

2. Thoren, K. L. \& Krantz, B. A. The unfolding story of anthrax toxin translocation. Mol. Microbiol. 80, 588-595 (2011).

3. Santelli, E., Bankston, L. A., Leppia, S. H. \& Liddington, R. C. Crystal structure of a complex between anthrax toxin and its host cell receptor. Nature 430, 905 (2004).

4. Kintzer, A. F. et al. The protective antigen component of anthrax toxin forms functional octameric complexes. J. Mol. Biol. 392, 614-629 (2009).

5. Mogridge, J., Cunningham, K. \& Collier, R. J. Stoichiometry of anthrax toxin complexes. Biochemistry 41, 1079-1082 (2002).

6. C. Antoni et al., Cryo-EM structure of the fully-loaded asymmetric anthrax lethal toxin in its heptameric pre-pore state. bioRxiv (2020).

7. Abrami, L., Liu, S., Cosson, P., Leppla, S. H. \& van der Goot, F. G. Anthrax toxin triggers endocytosis of its receptor via a lipid raft-mediated clathrin-dependent process. J. Cell Biol. 160, 321-328 (2003).

8. Miller, C. J., Elliott, J. L. \& Collier, R. J. Anthrax protective antigen: prepore-to-pore conversion. Biochemistry 38, 10432-10441 (1999).

9. Krantz, B. A., Finkelstein, A. \& Collier, R. J. Protein translocation through the anthrax toxin transmembrane pore is driven by a proton gradient. J. Mol. Biol. 355, 968-979 (2006).

10. Duesbery, N. S. et al. Proteolytic inactivation of MAP-kinase-kinase by anthrax lethal factor. Science 280, 734-737 (1998).

11. Feld, G. K. et al. Structural basis for the unfolding of anthrax lethal factor by protective antigen oligomers. Nat. Struct. Mol. Biol. 17, 1383-1390 (2010).

12. Brown, M. J., Thoren, K. L. \& Krantz, B. A. Role of the a clamp in the protein translocation mechanism of anthrax toxin. J. Mol. Biol. 427, 3340-3349 (2015).

13. Krantz, B. A. et al. A phenylalanine clamp catalyzes protein translocation through the anthrax toxin pore. Science 309, 777-781 (2005).

14. Jiang, J., Pentelute, B. L., Collier, R. J. \& Zhou, Z. H. Atomic structure of anthrax PA pore elucidates toxin translocation. Nature 521, 545 (2015).

15. Wynia-Smith, S. L., Brown, M. J., Chirichella, G., Kemalyan, G. \& Krantz, B. A. Electrostatic ratchet in the protective antigen channel promotes anthrax toxin translocation. J. Biol. Chem. 287, 43753-43764 (2012). 
16. Feld, G. K., Brown, M. J. \& Krantz, B. A. Ratcheting up protein translocation with anthrax toxin. Protein Sci. 21, 606-624 (2012).

17. Machen, A. et al. Asymmetric cryo-EM structure of anthrax toxin protective antigen pore with lethal factor N-terminal domain. Toxins 9, 298 (2017).

18. Hardenbrook, N. J. et al. Atomic structures of anthrax toxin protective antigen channels bound to partially unfolded lethal and edema factors. Nat. Commun. 11, 1-10 (2020).

19. Akkaladevi, N. et al. Assembly of anthrax toxin pore: Lethal-factor complexes into lipid nanodiscs. Protein Sci. 22, 492-501 (2013).

20. Akkaladevi, N. et al. Following natures lead: on the construction of membrane-inserted toxins in lipid bilayer nanodiscs. J. Membr. Biol. 248, 595-607 (2015).

21. Gogol, E. et al. Three dimensional structure of the anthrax toxin translocon-lethal factor complex by cryo-electron microscopy. Protein Sci. 22, 586-594 (2013).

22. Katayama, H. et al. Three-dimensional structure of the anthrax toxin pore inserted into lipid nanodiscs and lipid vesicles. Proc. Natl. Acad. Sci. 107, 3453-3457 (2010).

23. Ma, P., Cardenas, A. E., Chaudhari, M. I., Elber, R. \& Rempe, S. B. The impact of protonation on early translocation of anthrax lethal factor: Kinetics from molecular dynamics simulations and milestoning theory. J. Am. Chem. Soc. 139, 14837-14840 (2017).

24. M. T. Fisher, S. Naik (2019) Systems and methods for identifying protein stabilizers. (Google Patents).

25. Bayburt, T. H. \& Sligar, S. G. Membrane protein assembly into Nanodiscs. FEBS Lett. 584, 1721-1727 (2010).

26. Denisov, I., Grinkova, Y., Lazarides, A. \& Sligar, S. Directed self-assembly of monodisperse phospholipid bilayer Nanodiscs with controlled size. J. Am. Chem. Soc. 126, 3477-3487 (2004).

27. Ritchie, T. et al. Chapter eleven-reconstitution of membrane proteins in phospholipid bilayer nanodiscs. Methods Enzymol. 464, 211-231 (2009).

28. Krantz, B. A., Trivedi, A. D., Cunningham, K., Christensen, K. A. \& Collier, R. J. Acid-induced unfolding of the amino-terminal domains of the lethal and edema factors of anthrax toxin. J. Mol. Biol. 344, 739-756 (2004).

29. F. Wruck et al., The ribosome modulates folding inside the ribosomal exit tunnel. BioRxiv (2020).

30. Sun, J. \& Jacquez, P. Roles of anthrax toxin receptor 2 in anthrax toxin membrane insertion and pore formation. Toxins 8, 34 (2016).

31. Lacy, D. B., Wigelsworth, D. J., Melnyk, R. A., Harrison, S. C. \& Collier, R. J. Structure of heptameric protective antigen bound to an anthrax toxin receptor: a role for receptor in $\mathrm{pH}$-dependent pore formation. Proc. Natl. Acad. Sci. USA 101, 13147-13151 (2004).

32. Zhang, S., Finkelstein, A. \& Collier, R. J. Evidence that translocation of anthrax toxin's lethal factor is initiated by entry of its $\mathrm{N}$ terminus into the protective antigen channel. Proc. Natl. Acad. Sci. 101, 16756-16761 (2004).

33. Gupta, P., Singh, S., Tiwari, A., Bhat, R. \& Bhatnagar, R. Effect of $\mathrm{pH}$ on stability of anthrax lethal factor: correlation between denaturation and activity. Biochem. Biophys. Res. Commun. 284, 568-573 (2001).

34. Gonti, S., Westler, W. M., Miyagi, M. \& Bann, J. G. Site-specific labeling and $19 \mathrm{f}$ nmr provide direct evidence for dynamic behavior of the anthrax toxin pore $\phi$-clamp structure. Biochemistry 60, 643-647 (2021).

35. Das, D. \& Krantz, B. A. Peptide-and proton-driven allosteric clamps catalyze anthrax toxin translocation across membranes. Proc. Natl. Acad. Sci. 113, 9611-9616 (2016).

36. Das, D. \& Krantz, B. A. Secondary Structure Preferences of the Anthrax Toxin Protective Antigen Translocase. J. Mol. Biol. 429, 753-762 (2017).

37. Dmochewitz, L. et al. Role of CypA and Hsp90 in membrane translocation mediated by anthrax protective antigen. Cell. Microbiol. 13, 359-373 (2011).

38. Wilson, D. N. \& Beckmann, R. The ribosomal tunnel as a functional environment for nascent polypeptide folding and translational stalling. Curr. Opin. Struct. Biol. 21, 274-282 (2011).

39. Knapp, O. et al. Residues involved in the pore-forming activity of the $\mathrm{C}$ lostridium perfringens iota toxin. Cell. Microbiol. 17, 288-302 (2015).

40. Neumeyer, T. et al. Clostridium botulinum C2 Toxin identification of the binding site for chloroquine and related compounds and influence of the binding site on properties of the c2ii channel. J. Biol. Chem. 283, 3904-3914 (2008).

41. De, S. \& Olson, R. Crystal structure of the Vibrio cholerae cytolysin heptamer reveals common features among disparate poreforming toxins. Proc. Natl. Acad. Sci. 108, 7385-7390 (2011).

42. Piper, S. J. et al. Cryo-EM structures of the pore-forming A subunit from the Yersinia entomophaga ABC toxin. Nat. Commun. 10, 1952 (2019).

43. Yamada, T. et al. Cryo-EM structures reveal translocational unfolding in the clostridial binary iota toxin complex. Nat. Struct. Mol. Biol. 27, 288-296 (2020).

44. Sugawara, T. et al. Structural basis for pore-forming mechanism of staphylococcal a-hemolysin. Toxicon 108, 226-231 (2015).

45. Wigelsworth, D. J. et al. Binding stoichiometry and kinetics of the interaction of a human anthrax toxin receptor, CMG2, with protective antigen. J. Biol. Chem. 279, 23349-23356 (2004).

46. A. J. Machen et al., Analyzing dynamic protein complexes assembled on and released from biolayer interferometry biosensor using mass spectrometry and electron microscopy. JoVE, e57902 (2018).

47. Mastronarde, D. N. Automated electron microscope tomography using robust prediction of specimen movements. J. Struct. Biol. 152, 36-51 (2005).

48. Punjani, A., Rubinstein, J. L., Fleet, D. J. \& Brubaker, M. A. cryoSPARC: algorithms for rapid unsupervised cryo-EM structure determination. Nat. Methods 14, 290-296 (2017).

49. Zheng, S. Q. et al. MotionCor2: anisotropic correction of beam-induced motion for improved cryo-electron microscopy. Nat. Methods 14, 331-332 (2017).

50. Terwilliger, T. C., Ludtke, S. J., Read, R. J., Adams, P. D. \& Afonine, P. V. Improvement of cryo-EM maps by density modification. Nat. Methods 17, 923-927 (2020).

51. Adams, P. D. et al. PHENIX: a comprehensive Python-based system for macromolecular structure solution. Acta Crystallogr. D Biol. Crystallogr. 66, 213-221 (2010).

52. Pettersen, E. F. et al. UCSF Chimera-A visualization system for exploratory research and analysis. J. Comput. Chem. 25, 1605-1612 (2004).

53. Emsley, P. \& Cowtan, K. Coot: model-building tools for molecular graphics. Acta Crystallogr. D Biol. Crystallogr. 60, 2126-2132 (2004).

54. Chen, V. B. et al. MolProbity: All-atom structure validation for macromolecular crystallography. Acta Crystallogr. D Biol. Crystallogr. 66, 12-21 (2010).

\section{Acknowledgements}

This work is dedicated to the memory of our friend, mentor, and colleague Dr. Mark T. Fisher. Dr. Fisher was an integral in the vision, design, and implementation of this project. This work was supported by National Institutes of Health (R35-GM128562 and R03-AI142361 to B.D.F.), and by University of Kansas Madison and Lila Self Graduate Fellowship to A.J.M. The authors acknowledge the use of instruments at the Electron Imaging Center 
for NanoMachines supported by NIH (1S10RR23057 and 1S10OD018111), NSF (DBI-1338135) and CNSI at UCLA.

\section{Author contributions}

A.J.M., M.T.F., and B.D.F. designed the study. A.J.M. performed the experiments and data analysis. A.J.M. and B.D.F. wrote the manuscript.

\section{Competing interests}

The authors declare no competing interests.

\section{Additional information}

Supplementary Information The online version contains supplementary material available at https://doi.org/ 10.1038/s41598-021-91596-3.

Correspondence and requests for materials should be addressed to M.T.F. or B.D.F.

Reprints and permissions information is available at www.nature.com/reprints.

Publisher's note Springer Nature remains neutral with regard to jurisdictional claims in published maps and institutional affiliations.

Open Access This article is licensed under a Creative Commons Attribution 4.0 International License, which permits use, sharing, adaptation, distribution and reproduction in any medium or format, as long as you give appropriate credit to the original author(s) and the source, provide a link to the Creative Commons licence, and indicate if changes were made. The images or other third party material in this article are included in the article's Creative Commons licence, unless indicated otherwise in a credit line to the material. If material is not included in the article's Creative Commons licence and your intended use is not permitted by statutory regulation or exceeds the permitted use, you will need to obtain permission directly from the copyright holder. To view a copy of this licence, visit http://creativecommons.org/licenses/by/4.0/.

(C) The Author(s) 2021 